\title{
ANCHOR DENSITY MINIMIZATION FOR LOCALIZATION IN WIRELESS SENSOR NETWORK (WSN)
}

\author{
Nour Zaarour, Nadir Hakem and NahiKandil \\ Engineering School, UQAT-LRTCS, Rouyn-Noranda, Canada
}

\begin{abstract}
In wireless sensor networks (WSN) high-accuracy localization is crucial for both of WNS management and many other numerous location-based applications. Only a subset of nodes in a WSN is deployed as anchor nodes with their locations a priori known to localize unknown sensor nodes. The accuracy of the estimated position depends on the number of anchor nodes. Obviously, increasing the number or ratio of anchors will undoubtedly increase the localization accuracy. However, it severely constrains the flexibility of WSN deployment while impacting costs and energy. This paper aims to drastically reduce anchor number or ratio of anchor in WSN deployment and ensures a good trade-off for localization accuracy. Hence, this work presents an approach to decrease the number of anchor nodes without compromising localization accuracy. Assuming a random string WSN topology, the results in terms of anchor rates and localization accuracy are presented and show significant reduction in anchor deployment rates from $32 \%$ to $2 \%$.
\end{abstract}

\section{KEYWORDS}

Wireless sensor network (WSN), anchors, received signal strength (RSS), localization, path-loss exponent (PLE), connectivity.

\section{INTRODUCTION}

Localization in wireless sensor networks (WSN) is anessential and critical issue. Most WSN applications necessitate the location of the sensor nodes such as in environment surveillance, object tracking, emergency services, asset management, location-based recommendations, and geosocial networks [1] [2]. Knowing the location is not only necessary to identify the geographic origin of events, for example, the location of a fire or the location of the enemy on a battlefield for the deployment of troops, but it can help in various functionalities system, such as geographic routing, network coverage, perimeter search, topology control, and location-based information polling. Moreover, the availability of cheap wireless networks and the surge in adoption of smartphones make the location-based services (LBS) omnipresent. Indoor LBSs promise enormous potential for research organizations to adapt to different indoor applications such as emergency services and assisted health care systems [2].

One of the simplest techniques is to locate the nodes manually when they are deployed in the environment. However, manual localization is costly in time, due to the large number of nodes to be located. Another technique is to use the Global Positioning System (GPS) which provides highly accurate location information, but it may not be feasible for most WSN deployments such as indoor environment deployment [3]. 
Since sensor nodes are energy constrained, solutions like GPS are not recommended, GPS components available for WSNs are very costly, exceeding almost three times the cost of a sensor node [4]. Likewise, in some hostile or indoor environments, GPS performance will deteriorate significantly and therefore will be unreliable for location [5]. Hence, various techniques and localization algorithms have been proposed in the literature to localize sensors in WSN [6] [7] [8], however, to achieve high accuracy these techniques, a high percentage of anchors whose location relative to a global reference axis are known a priori has to be used. Nevertheless, not every node of deployed WSN can be equipped with localization components, and that due to cost and power consumption reasons. In this work, we present a localization approach aiming to decrease anchor density, hence network cost, while maintaining a high localization accuracy. This is achieved by using the joint parameter and distance estimation approach based on connectivity and received signal strength.

The remainder of this paper is organized as follows: related works and anchor density impact are discussed in section 2. In section 3, the joint parameter and distance estimation approach based on connectivity- RSS is summarized. Localization and simulations results are analyzed in section 4 , and a conclusion and perspectives are drawn in section 5 .

\section{RELATED WORKS}

\subsection{Basic Localization Methods}

Localization techniques are classified in different categories. Figure 1 presents the taxonomy of localization techniques. An anchor-based localization algorithm uses one or more anchors. These nodes provide location information, in the form of beacon messages, to other nodes whose position is unknown so that they can be located, forming a global coordinate system where the location of each node is estimated, hence the localization is absolute. However, in an anchor free technique, the sensors cooperate with their neighbors, without the use of anchors, and form a local coordinate system where the location of each node is estimated, hence a relative localization is [7].

In centralized techniques, anchors collect the measurements of the unknown node to localize and then send them to a central processor to calculate the position of the unknown node. Usually, this type is not very scalable, as the aggregation of required information such as anchor locations and metrics can require many node collaborations, causing unnecessary overhead and even congestion. While, in distributed techniques the target node can only infer its own location based on information collected locally, and independently.

Range-based localization technique uses the measured distance/angle between nodes to estimate the location. Common measurements used for localizing nodes in WSNs are the RSS [7], time of arrival (ToA) [8], time difference of arrival (TDoA) [9], angle of arrival (AoA) [10]. However, range-free localization technique uses the connectivity or pattern matching method to estimate the location. Such as the approximate point-in-triangulation test (APIT) algorithm [12], the distance vector-hop (DV-Hop) algorithm [13], the centroid localization algorithm [11]. The advantage of using range-based techniques is that they have a high accuracy range compared to range-free techniques. However, these techniques are limited because they require additional hardware, which is expensive for large systems. While in range-free techniques, it is not necessary to determine distances directly; instead, they use radio connectivity to calculate the number of hops between nodes and estimate the location using geometry methods. Certain advantages can be obtained by using these techniques which do not require special hardware support; generally, they are cost effective, mainly to the detriment of the level of precision [14]. 
The fingerprinting technique or scene analysis is another branch of localization technique. It uses the signatures, and is based on a study campaign conducted in the environment where the location system works. In this method the signal characteristics obtained from a set of locations are catalogued in a first phase, called off-line phase, aiming to build the signature database. Several types of signatures [23] can be used: the powers, angles of arrival, arrival time, broadband parameters such effective delay spread or the number of reflected paths of signals received from the fixed base stations. In the second phase called the real time phase, the locations of the node are estimated by comparing the nodes current signal characteristics with those catalogued previously. However, the requirement for generating a signal signature database makes this technique a laborious collection of data during scene analysis or even unachievable for the most scenarios of the WSNs especially in complex environments.

RSS-based methods are ideal for low-cost and low complexity networks, since no additional hardware is needed. However, the exact knowledge of the propagation model is of greatest importance for RSS-based localization or ranging. A previous work presented a hybrid approach which uses the information of the range-free technique (connectivity information) in order to rectify the errors obtained by the range-based method and that by estimating the parameters of the propagation model to better map RSS measurements into inter-node distance estimation [15] however, the latter work does not shed light on keeping high localization accuracy with a low anchor density.

The accuracy of localization technique is greatly affected by the number of anchors and their placement, playing an essential role in the cost of the network. Many studies have investigated optimal number and placement of anchors to increase the localization accuracy [16] [17] [18]. Moreover, they study optimal anchor placement in area-based localization algorithms with the goal of providing the best placement that maximises accuracy. However, to the best of our knowledge, no work has a goal to decrease the number of anchor while keeping high localization accuracy. Hence, the aim of this paper is to present a localization approach with a low anchor density and a high localization accuracy.

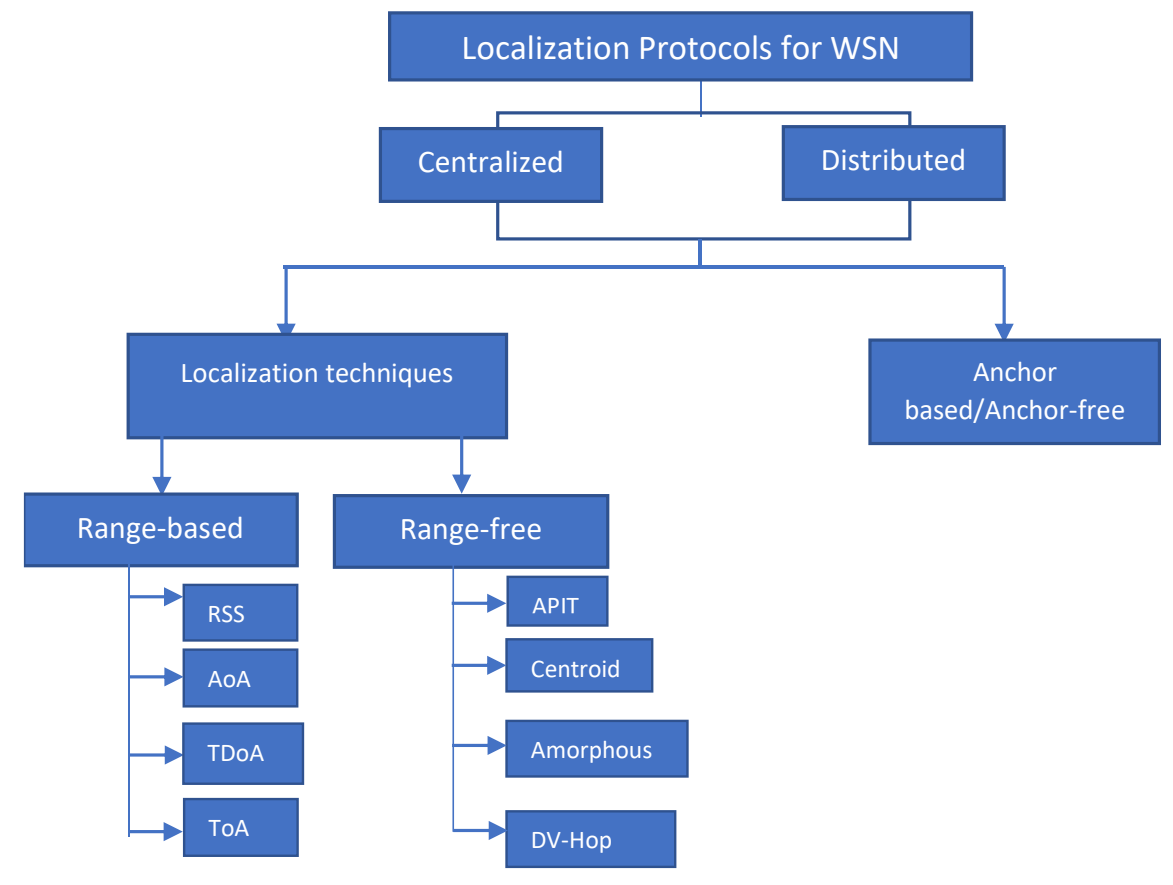

Figure 1. Taxonomy of localization techniques 


\subsection{Sensor node location}

Given a distance measurement between a sensor node and an anchor node, the position of unknown node must be along the circumference of a circle (in two-dimensional space) or sphere (in three-dimensional space) centered at the reference node, with the radius representing the distance between the reference node and the sensor node. At least two reference nodes in one dimension, three non-collinear reference nodes in two dimensions, or four non-coplanar reference nodes in three dimensions are required to obtain a unique location. This process is called trilateration, which assumes perfect distance measurements, which is not achievable in WSNs because of ranging errors.

\subsection{Anchor Density Effect}

The accuracy of a localization method is governed not only by the efficiency of distance estimation between unknown and anchor node, but also by the number (accuracy increases with anchor percentage) and the position of the anchors themselves [19]. Researchers working on anchor based WSN localization have always been interested in the effect of number and placement of anchor nodes in the network [20]. They have focused on reducing the position error introduced by placement and percentage of anchor nodes in the network. Localization error decreases with the increasing of connected anchor nodes. However, increasing anchor nodes will increase the cost of the deployed network. The main aim in this work is to reduce anchor nodes' percentage while assuring a high localization accuracy.

\section{Connectivity-Based Joint Parameter Estimation}

This section summarizes the approach used to reduce anchor density which proposes a joint estimation scheme for the range, path-loss exponent (PLE), and inter-node distances based on the received signal strength (RSS) and the network's information [15].

\subsection{Assumptions}

Consider a homogeneous Poisson point process (PPP) in a one-dimensional WSN consisting of $N$ nodes placed randomly at positions $x_{i}$ for $i=1, \ldots, N$ along the deployment segment $\left[x_{\min } x_{\max }\right]$, with node density $\lambda=\frac{N}{\left(x_{\max }-x_{\min }\right)}$, and having transmission ranges $R_{i}$ for $i=$ $1, \ldots, N$. This topology is well-justified in environments that impose one-dimensional deployments such as narrow-vein underground mines [21], sewage or water distribution networks, etc. The received signal power in $\mathrm{dBm}$ is modeled as the sum of large-scale path-loss and $\log$ - Normal shadowing. The received power $P r_{i j}$ at node $i$ of a signal emitted from node $j$ is modeled by [22] as determined in equation 1 .

$$
\operatorname{Pr}_{i j}(d i j)=\operatorname{Pr}\left(d_{0}\right)-10 \gamma \log \left(\frac{d_{i j}}{d_{0}}\right)+X_{\sigma}
$$

Where $\operatorname{Pr}\left(d_{0}\right)$ is the received power from any given node at the reference distance $d_{0}=1, \gamma$ is the PLE with common values ranging between 2 and $6, d_{i j}$ is the distance separating the two nodes $i$ and $j$, and $X_{\sigma}$ is the large-scale log-Normal shadowing with variance $\sigma^{2}$.

\subsection{Poisson Point Process (PPP)}

A uniform (homogeneous) PPP is defined in [24] as: 
"Let $\Lambda$ be a locally finite measure on some metric space E. A point processes $\Phi$ is Poisson on $E$ if

- For all disjoint subsets $A_{1}, \cdots, A_{n}$ of $E$, the random variables $\Phi\left(A_{i}\right)$ are independent

- For all sets $A$ of $E$, the random variables $\Phi(A)$ are Poisson”

If a Poisson point process has a constant parameter, $\lambda$, then it is considered a homogeneous or stationary PPP [25]. In fact, the parameter $\lambda$ can be interpreted as the average number of points per unit of length, area or volume, so it is sometimes referred to as the average density.

If two real numbers $a$ and $b$, such as $a \leq b$, representing points in time, belong to a PPP with parameter $\lambda>0$, then the probability of $n$ points existing in the interval $(a, b]$ is given by equation 2.

$$
P\{N(a, b]=n\}=\frac{[\lambda(b-a)]^{n}}{n !} e^{-\lambda(b-a)}
$$

\subsection{Connectivity Information}

Two nodes are neighbors at one hop if they are connected, hence, $C_{i j}$ is a random variable presenting the connectivity information defined as in equation 3 .

$$
C_{i j}=\left\{\begin{array}{l}
1 \text { if } P r_{i j} \geq P_{t h} \\
0 \text { if } P r_{i j}<P_{t h}
\end{array}\right.
$$

Where $P_{t h}$ is the power detection threshold.

\subsection{Proposed Estimation}

\subsubsection{PLE estimation}

The estimated PLE, $\hat{\bar{\gamma}}$, over the entire wireless sensor network will be estimated by equation 4 .

$$
\hat{\bar{\gamma}}=\frac{1}{N} \sum_{i=1}^{N} \hat{\gamma}_{i}
$$

Where, $\hat{\gamma}_{i}, \hat{R}_{i}, \hat{\bar{R}}$ are determined in equations 5,6 , and 7 respectively.

$$
\begin{gathered}
\hat{\gamma}_{i}=\frac{-P_{t h}+P_{r}\left(d_{0}\right)}{10 \log _{10}\left(\hat{R}_{i}\right)} \\
\hat{R}_{i}=\frac{1}{2 \lambda} \sum_{j=1}^{N} C_{i j}
\end{gathered}
$$

And

$$
\hat{R}=\frac{1}{N} \sum_{i=1}^{N} \hat{R}_{i}
$$




\subsubsection{Distance estimation}

Each node $i$, for $i=1, \ldots, N$, estimates its distances to its connected neighbor nodes $k \neq i$ as in equation (8).

$$
\hat{d}_{i k}=10^{\frac{P_{r}\left(d_{0}\right)-P r_{i k}}{10} \hat{\gamma}}
$$

Where $\operatorname{Pr}_{\mathrm{ik}}$ is the received power at node $i$ from node $k$.

\section{Localization AND SimUlations RESUltS}

\subsection{Assumptions and WSN Model}

The approach presented in this paper consists in decreasing the numberof anchor nodes. To prove its efficiency, a multi-hop linear WSN of $N$ nodes is considered, it is deployed in a homogeneous environment, i.e., all nodes have a priori the same communication range $R_{i}=R$ for $i=$ $1, \ldots, N$ with density $\lambda$. Nodes are positioned in linear topology, on a distance $d=x_{\max }-x_{\min }$ as shown in figure 2 . However, possible extensions to $2 D$ or $3 D$ network topologies, beyond the scope of this contribution, are currently under investigation and will be addressed in future publications. Its normalized error (NE), $\varepsilon_{x}$, is assessed as computed in equation 9.

$$
\varepsilon_{x}=\frac{\left|\left(x_{i}-\hat{x}_{i}\right)\right|}{x_{i}}
$$

Where $x_{i}$ is the position in one dimension of a node $i, i=1, \ldots, N$, and $\hat{x}_{i}$ is its estimated position.

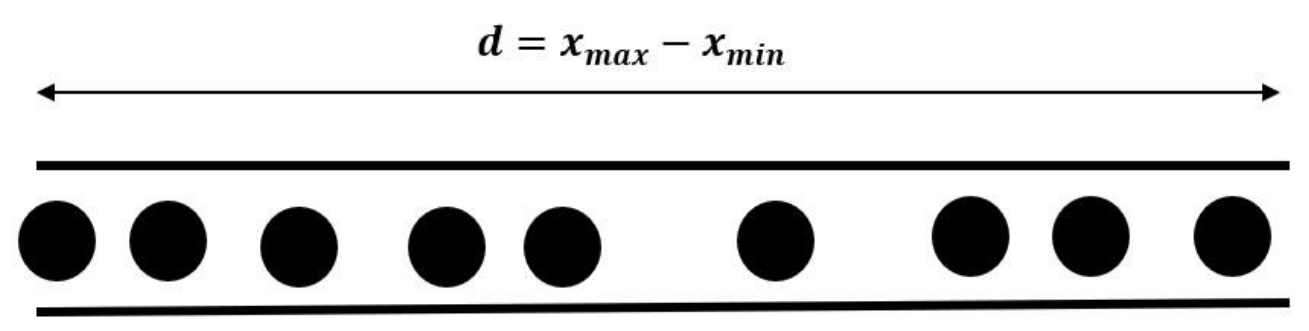

Figure 2. WSN Topology

\subsection{Results and Analysis}

Extensive simulations are conducted to show the efficiency of the proposed approach, where 1000 topology are randomly generated following the Poisson distribution. Moreover, this is done for different values of PLE and $\sigma$, the log-Normal shadowing standard deviation. Simulations are done using MATLAB. All relevant simulation parameters are listed in Table 1. 
Table 1. WSN Simulation Parameters Setup

\begin{tabular}{|l|l|}
\hline Parameter (Unit) & Value (s) \\
\hline$\gamma:$ PLE & $(3 ; 4)$ \\
\hline$N:$ Sensor set cardinality & 100 \\
\hline $\begin{array}{l}\lambda: \text { WSN density (average distance } \\
\text { between 2 adjacent sensors) (node/m) }\end{array}$ & $1 / 3$ \\
\hline $\begin{array}{l}\sigma: \text { Log-Normal shadowing standard } \\
\text { deviation }(\mathrm{dB})\end{array}$ & $(1 ; 2 ; 3 ; 4 ; 5 ; 6)$ \\
\hline $\begin{array}{l}\operatorname{Pr}\left(d_{0}\right): \text { Received power at reference } \\
d_{0}=1(\mathrm{dBm})\end{array}$ & -45 \\
\hline$P_{t h}:$ Threshold power $(\mathrm{dBm})$ & -90 \\
\hline Anchor number & $(32 ; 8 ; 2)$ \\
\hline Number of topologies & 1000 \\
\hline
\end{tabular}

Positions of anchors are chosen to cover the deployed network, with a step $\eta$ such as in equation 10.

$$
\eta=\frac{x_{\max }-x_{\min }}{N_{A}}
$$

Where $N_{A}$ is the number of anchors, unknown position is estimated using multilateration or bilateration in a one -dimensional deployment.

Figures 3 and 4 present the cumulative density function (CDF) of normalized localization error for $\gamma=3$ for both unknown homogeneous and known homogenous environment respectively for different values of anchor number. With the proposed strategy, until $90 \%$ of the sensors could estimate their position with a NE less than 0.04 while using 32 anchors which represent $32 \%$ of total node number in an unknown homogeneous WSN. In contrast, 78\% of sensors achieve the same accuracy with only 2 anchors, when the WSN is unknown homogeneous a priori. However, $90 \%$ of sensors estimate positions with NE equals to 0.1 with 2 anchors.

On the other hand, $90 \%$ of the sensors estimate their position with a NE less than 0.01 while using 32 anchors, while $82 \%$ of sensors achieve the same accuracy with only 2 anchors in a known homogenous WSN. On the other hand, $90 \%$ of sensors estimate the position with an error equal to 0.018 with 2 anchors.

Likewise, figures 5 and 6 present $\mathrm{CDF}$ of normalized localization error for $\gamma=4$. Results in figure 5 where the WSN is unknown homogeneous show that until $90 \%$ of the sensors could estimate their position with a NE less than 0.012 using 32 anchors, this percentage decreases to $83 \%$ while using only 2 anchors. Moreover, $90 \%$ of the sensors could estimate positions with a NE less than 0.02 with 2 anchors. Also, figure 6 shows the same results as $90 \%$ of sensors achieve an error of 0.03 with 32 anchors, and $76 \%$ of sensors achieve this error with 2 anchors in a known homogenous WSN.

Results obtained show efficiency of the technique in using less anchors while maintaining high localization accuracy. In the example used in this case the anchor's number is passing from using 32 anchors to only 2 anchors with a little increase in error values, $\Delta N E=0.006$ for $\gamma=3$ in a priori unknown homogeneous WSN, $\Delta N E=0.008$ for $\gamma=3$ in a known homogeneous WSN, $\Delta N E=0.008$ for $\gamma=4$ in a priori known homogeneous WSN and $\Delta N E=0.018$ for $\gamma=4$ in an unknown homogeneous WSN. Decreasing anchor nodes will decrease network cost which is an important constraint in WSN. In addition to, it can be observed that in homogeneous network the 
localization errors are less than those obtained in an unknown homogeneous network. This shows the advantage of knowing a priori that a WSN is homogeneous., i.e., nodes have a priori the same communication range $R_{i}$.

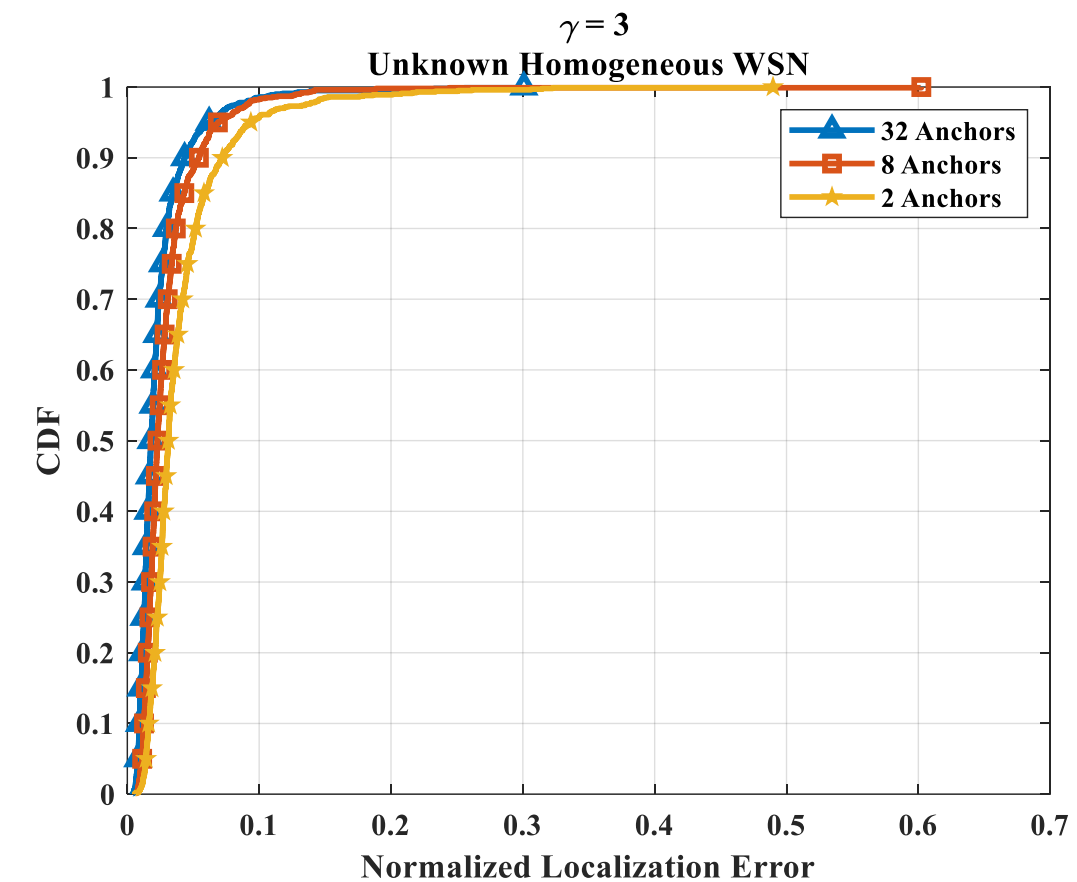

Figure 3. $\mathrm{CDF}$ of Normalized Error for PLE $=3$ in an Unknown Homogenous WSN

$\gamma=\mathbf{3}$

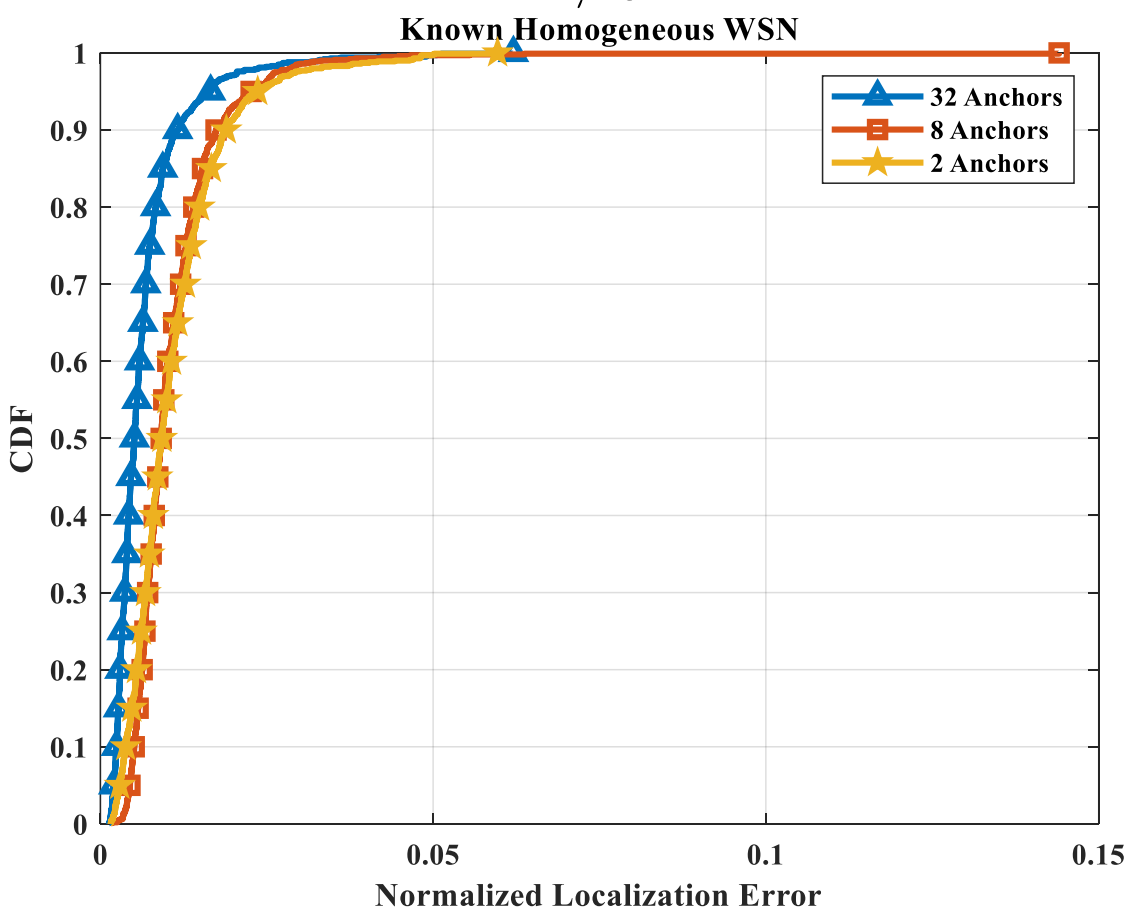

Figure 4. CDF of Normalized Error for PLE=3 in a Known Homogenous WSN 


$$
\gamma=4
$$

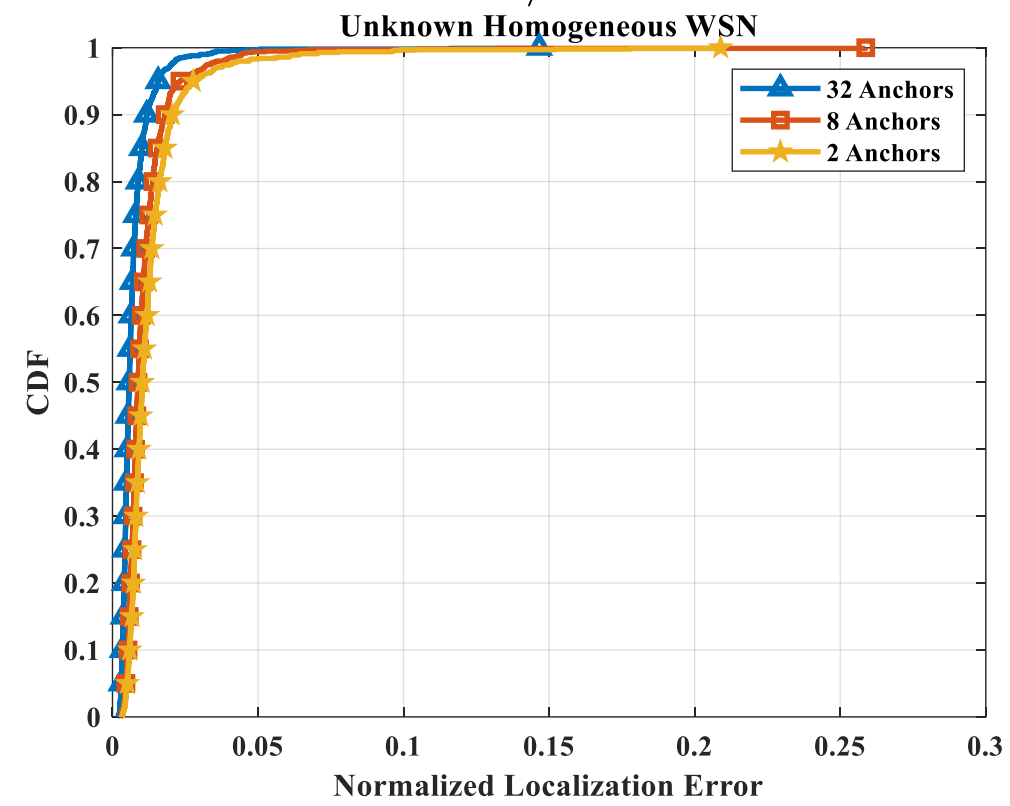

Figure 5. CDF of Normalized Error for PLE=4 in an Unknown Homogenous WSN

$$
\gamma=4
$$

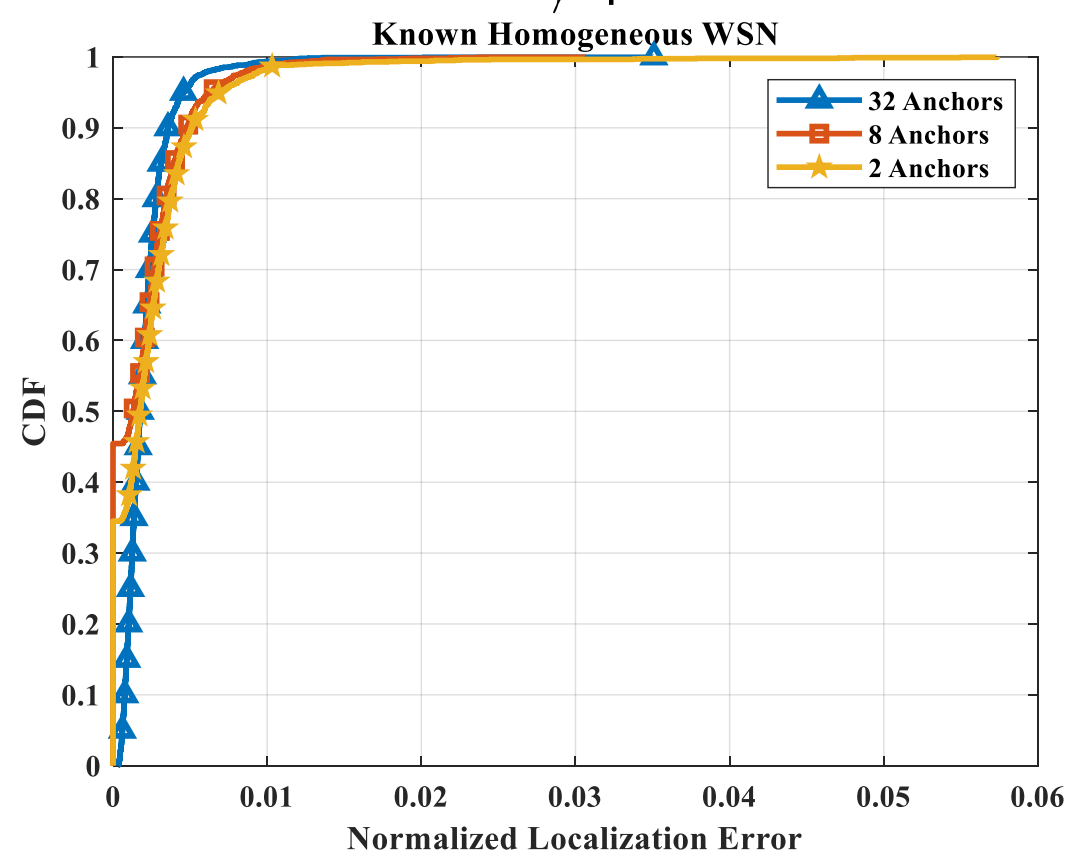

Figure 6. $\mathrm{CDF}$ of Normalized Error for $\mathrm{PLE}=4$ in a Known Homogenous WSN

\section{Conclusions}

In this paper an anchor number optimization in localization in WSN is presented. By using the approach based on connectivity and network information, the method is able to localize sensors in a WSN with a very low number of anchors and with high accuracy. Hence, the efficiency of the proposed approach based on estimating channel properties to compensate the anchor number in 
the localization process is proved. Indeed, in terms of anchor rates results show reduction in anchor deployment rates from $32 \%$ to $2 \%$. This solution was derived for one-dimensional WSNs used in many new applications. However, extensions to two- or three-dimensional network topologies are under investigation. Also, other deployment assumptions are under investigation such as gaussian deployment.

\section{REFERENCES}

[1] Wang, Zengfeng; Zhang, Hao; Lu, Tingting\& Gulliver, T. Aaron, (2018) “A Grid-Based Localization Algorithm for Wireless Sensor Networks Using Connectivity and RSS Rank,"in IEEE Access, vol. 6, pp. 8426-8439.

[2] Muhammad Aamir, Cheema, (2018) "Indoor location-based services: challenges and opportunities", SIGSPATIAL Special, Volume 10, Issue 2, pp 10-17.

[3] Ian F. Akyildiz \& Mehmet Can Vuran, (2010) Wireless Sensor Networks. John Wiley Sons Ltd.

[4] Vijayalakshmi S. R \& Muruganand, (2018) Wireless Sensor Networks: Architecture, Applications, Advancements. Mercury Learning and Information: Dulles, Virginia.

[5] Leila, Chelouah; Fouzi, Semchedine \& Louiza Bouallouche-Medjkoune, (2018) "Localization protocols for mobile wireless sensor networks: A survey", Computers \& Electrical Engineering, Volume 71, pages 733-751.

[6] Neha Sharma \& Vishal Gupta, (2020) "Meta-Heuristic-Based Optimization of WSNs Localisation Problem- a Survey", Procedia Computer Science, Volume 173, Pages 36-45.

[7] Tomic, Slavisa; Beko, Marko \& Dinis, Rui, (2015) "RSS-Based Localization in Wireless Sensor Networks Using Convex Relaxation: Noncooperative and Cooperative Schemes," IEEE Transactions on Vehicular Technology, vol. 64, no. 5, pp. 2037-2050.

[8] Xu, Enyang; Ding, Zhi \& Dasgupta, Soura, (2011) "Source Localization in Wireless Sensor Networks from Signal Time-of-Arrival Measurements,” IEEE Transactions on Signal Processing, vol. 59, no. 6, pp. 2887-2897.

[9] Zhao, Yue; Li, Zan; Hao, Benjian \& Shi, Jia, (2019) "Sensor Selection for TDOA-Based Localization in Wireless Sensor Networks with Non-Line-of-Sight Condition," in IEEE Transactions on Vehicular Technology, vol. 68, no. 10, pp. 9935-9950.

[10] Shao, Hua-Jie; Zhang, Xiao-Ping \& Wang, Zhi, (2014) "Efficient Closed-Form Algorithms for AOA Based Self-Localization of Sensor Nodes Using Auxiliary Variables," in IEEE Transactions on Signal Processing, vol. 62, no. 10, pp. 2580-2594.

[11] $\mathrm{Ru}$, Lulu \& Zhang, Linghua, (2017) "A weighted Centroid Localization Algorithm for Wireless Sensor Networks Based on Weight Correction," 2017 9th International Conference on Advanced Infocomm Technology (ICAIT), pp. 165-169.

[12] Wang \& Jin H. (2009) "Improvement on APIT localization algorithms for wireless sensor networks," in Proceedings of the International Conference on Networks Security, Wireless Communications and Trusted Computing (NSWCTC '09), pp. 719-723.

[13] Li, Xiaoyin; Yan, Lianshan; Pan, Wei \& Luo, Bin (2017) "Secure and Robust DV-Hop Localization Based on the Vector Refinement Feedback Method for Wireless Sensor Networks," in The Computer Journal, vol. 60, no. 6, pp. 810-821.

[14] Ibrahim, Nemer; Tarek, Sheltami; Elhadi, Shakshuki; Abdulrahman, Abu Elkhail\&Mumin Adam, (2021) Performance evaluation of range-free localization algorithms for wireless sensor networks. Pers UbiquitComput 25, pp. 177-203.

[15] Nour, Zaarour; Sofiène, Affes; Nahi, Kandil \& Nadir, Hakem, (2020) "Connectivity-Based Joint Parameter Estimation in One-Dimensional Wireless Sensor Networks," 2020 International Wireless Communications and Mobile Computing (IWCMC), pp. 358-364.

[16] Dai, Wenhan; Shen, Yuan\& Win, Moe Z Dai W, (2012) "On the minimum number of active anchors for optimal localization,” 2012 IEEE Global Communications Conference (GLOBECOM) pp. 49514956.

[17] Du, Shifeng; Huang, Baoqi; Jia, Bing \& Li, Wuyungerile, (2019) "Optimal Anchor Placement for Localization in Large-Scale Wireless Sensor Networks," IEEE 5th International Conference on Data Science and Systems (HPCC/SmartCity/DSS), pp. 2409-2415. 
[18] Lasla, Noureddine; Younis, Mohamed; Ouadjaout, Abdelraouf \& Badache, Nadjib, (2015) "On optimal anchor placement for efficient area-based localization in wireless networks," 2015 IEEE International Conference on Communications (ICC), pp. 3257-3262.

[19] Ammari, Habib M., (2014) The Art of Wireless Sensor Networks: Volume 1 Fundamentals. Springer Publishing Company, Incorporated.

[20] Savvides, A; Garber, W.L.; Moses, R.L. \& Srivastava, M.B, (2005) “An Analysis of Error Inducing Parameters in Multihop Sensor Node Localization". IEEE Transactions on Mobile Computing, 4:567-577.

[21] Shah, Kaushal \& Jinwala, Devesh C., (2016) "A Secure Expansive Aggregation in Wireless Sensor Networks for Linear Infrastructure,” in Proc. of IEEE Region 10 Symposium (TENSYMP), Bali, Indonesia, pp. 207-212.

[22] Rappaport T.S., (1999)Wireless Communications. New Jersey: Prentice Hall.

[23] Isabelle, Vin; Davy, P. Gaillot; Pierre, Laly; Martine, Liénard \& Pierre, Degauque, (2015) "Overview of Mobile Localization Techniques and Performances of a Novel Fingerprinting-Based Method", Comptes Rendus Physique, Volume 16, Issue 9, Pages 862-873.

[24] Haenggi, Martin; Andrews, Jeffrey G.; Baccelli, Francois; Dousse, Olivier \& Franceschetti, Massimo, (2009) "Stochastic geometry and random graphs for the analysis and design of wireless networks," in IEEE Journal on Selected Areas in Communications, vol. 27, no. 7, pp. 1029-1046.

[25] Jesper, Moller \& Rasmus, Plenge Waagepetersen, (2004) Statistical Inference and Simulation for Spatial Point Process, Chapman and Hall/CRC.

\section{AUTHORS}

Nour Zaarour: received the Bachelor's Degree in biomedical engineering from the Islamic University of Lebanon (IUL), Khaldeh, Lebanon in 2010, and the M.Sc. degree from the University of Quebec in Abitibi-Témiscamingue (UQAT), Val-d'Or, Qc, Canada, in 2013. She is currently pursuing the PhD. degree with the UQAT. Her research interests include channel modeling, localization algorithms, wireless sensor networks and neural networks.

Nadir Hakem is in charge of innovation and digital strategy for Meglab a subsidiary Epiroc company, Canada since May 2021. Previously, he was Professor at the School of Engineer from 2009 to 2021 and he has been a research assistant from 2006 to 2009 at the University of Québec Abitibi-Témiscamingue (UQAT). He is involved in research work in radio frequency measurements and analytical modeling, energy harvesting and localization issues for wireless sensor network.

Dr. Hakem obtained his Advanced Studies Diploma and his $\mathrm{PhD}$ in Computer

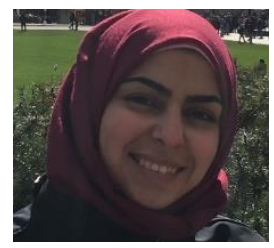
Science degree respectively in 1999 and 2004 both at Blaise Pascal University, France. He holds an engineer's degree in computer science from the science and Technology University of Algiers, Algeria. He is also a member of the Order of Engineers of the Province of Québec (OIQ) and the Institute of Electrical and Electronics Engineers (IEEE). He has been involved in many scientific conference committees and in supervision of over 30 graduate and post-graduate students.

NahiKandil is a professor at the School of Engineering in the University of Quebec in Abitibi-Timiskaming (UQAT) since 2000. He has worked on research dealing with neural networks and their applications to wireless communications and power systems. Prof. Kandil is now working mainly with localization and channel modeling in confined areas and mine environments.

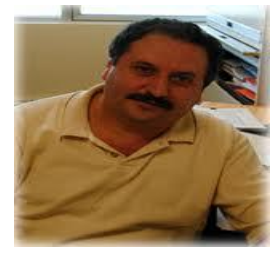

(C) 2021 By AIRCC Publishing Corporation. This article is published under the Creative Commons Attribution (CC BY) license. 